\title{
Fallopian tube carcinoma presenting as preterm labour at 3I weeks gestation woman
}

\begin{abstract}
Primary Fallopian tube carcinoma (PFTC) is a rare tumour accounting for less than $2 \%$ of all female genital malignancies. The true incidence of PFTC is underestimated due to difficulty to diagnose it as primary disease and is often mistaken for metastases from ovarian cancer. A 37-year-old woman presented with pre-term contractions in her 31 st week of gestation. Ultrasound scan confirmed a left ovarian mass and ascites. The Ca125 was $2450 \mathrm{IU} / 1$. Planned Caesarean section was performed at 34 weeks and a live female infant was born in good condition. At the same time she underwent a total abdominal hysterectomy, bilateral salpingo-oophorectomy, omentectomy and biopsy of pelvic peritoneum and appendices epiploicae. Over 6L ascites was drained. The histology confirmed Stage IIB adenocarcinoma of endometrioid type arising from the left fallopian tube with metastases to ovaries, peritoneum.
\end{abstract}

Keywords: adenocarcinoma, female genital malignancies, adnexal masses in pregnancy, ultrasound
Volume 2 Issue I - 2015

\author{
Wassim A Hassan,' 'Medhat Alberry,' Panayoti \\ Bachkangi, ${ }^{2}$ Clemens VonWidekind ${ }^{3}$ \\ 'Fetal Medicine Department, Cambridge University Hospitals \\ NHS Foundation Trust, UK \\ ${ }^{2}$ Department of Cancer Studies \& Molecular Medicine, \\ University of Leicester, Leicester, UK \\ ${ }^{3}$ Department of Obstetrics \& Gynaecology, Northampton \\ General Hospital, UK
}

\author{
Correspondence: Wassim A Hassan, Fetal Medicine \\ Department, Rosie Maternity Hospital, Box 225, Addenbrooke's \\ Hospital, Cambridge University Hospitals, CB2 2QQ, \\ Cambridge, United Kingdom, Tel 0044-7925595527, \\ Email wassim.hassan@me.com
}

Received: October 23, 2014 | Published: January 7, 2015
Abbreviations: PFTC, primary fallopian tube carcinoma; EOC, epithelial ovarian carcinoma; FIGO, International federation of gynecology and obstetrics; CA 125, cancer antigen 125 or carbohydrate antigen 125

\section{Introduction}

Primary Fallopian tube carcinoma (PFTC) is rare accounting for $0.14-1.8 \%$ of all female genital malignancies. ${ }^{1}$ It was first described by Renaud in 1847. In England and Wales 40 cases of PFTC are registered annually and few hundred cases are reported annually in the United States. The incidence of fallopian tube cancer is often underestimated due to its co-existence with ovarian masses, and frequently Fallopian tube carcinoma is misdiagnosed as a metastasis from a primary ovarian carcinoma. ${ }^{2}$ Cancers that metastasise to fallopian tubes include ovarian, endometrial, gastrointestinal tract and breast. $^{3}$

We describe a case of PFTC diagnosed in a 37 years old woman who presented with preterm contractions at 31 weeks gestation.

\section{Case presentation}

A 37-year-old multiparous woman presented at $30^{+6}$ weeks gestation with regular uterine contractions. She had uncomplicated past medical history and her pregnancy was straight forward to date. There was no history of preterm rupture of membranes, or loss per vaginum. She had no urinary symptoms or change in bowel habits. On examination, the patient was stable with normal vital signs. The baby was in longitudinal lie, breech presentation and measured appropriately for dates. On vaginal examination, the cervix was $2 \mathrm{~cm}$ long, not dilated and was displaced anteriorly and to the right by a solid mass in the pouch of Douglas. The patient had palpable uterine contractions and was managed according to our preterm labour protocol and was given a course of steroids and commenced on tocolytics. Later the contractions settled.

An ultrasound scan revealed a 179x129x90mm mixed echogenic mass in the pelvis, which risen from the left and deviated the cervix towards the right and anterior. Sonographic appearances were suggestive of a cystadenoma or cystadenocarcinoma. Ascites was noticed around the uterus and extending into the right and left upper quadrant, with the deepest pool measuring $90 \mathrm{~mm}$. The Ca125 was $2450 \mathrm{U} / \mathrm{ml}$. Other tumour markers including CEA and $\alpha$-feto protein were within normal limits. An MRI scan showed bilateral complex adnexal masses which likely represented ovarian neoplasm. High T1 signal changes in the right-sided lesion were noticed suggesting haemorrhage. No evidence of retroperitoneal lymph node involvement was noticed on MRI. A working diagnosis of epithelial ovarian carcinoma (EOC) was postulated. The case was discussed at a multidisciplinary team meeting (Obstetrics, Gynaecology-Oncology, Anaesthetics, Neonatology, Radiology and Medical Oncology); a consensus was reached for a planned delivery and debulking surgery at 34 weeks where adequate maturity of the fetus is achieved.

In the interim period, there was massive accumulation of ascites and the Ca125 level doubled to 5013IU/L. As planned, at 34 weeks gestation, a live female infant was delivered by Caesarean section in good condition. At the time of laparotomy, $6 \mathrm{~L}$ of ascites were drained. The right ovary measured $6 \mathrm{~cm}$ while the left ovary measured $14 \mathrm{~cm}$ and was adherent to posterior pelvic wall and sigmoid colon. There were multiple deposits of tumour on the uterine surface, appendices epiploicae, Pouch of Douglass, peritoneum, and anterior (pelvic) abdominal wall peritoneum. Total abdominal hysterectomy, bilateral salpingo-oophorectomy, infracolic omentectomy, biopsy of pelvic peritoneum and appendices epiploicae were performed. The residual disease included inflammatory plaque disease in the Pouch of Douglas of less than $1 \mathrm{~cm}^{3}$. 
Histology analysis of the specimen showed poorly differentiated adenocarcinoma of endometrioid type, arising from the left fallopian tube with metastatic spread to ovaries, peritoneum and the appendix epiploicae as shown in the Figures 1-3. No metastatic spreads were found in the omentum. The cytology of the ascites was positive for adenocarcinoma cells. FIGO staging was of fallopian tube tumour stage IIB.

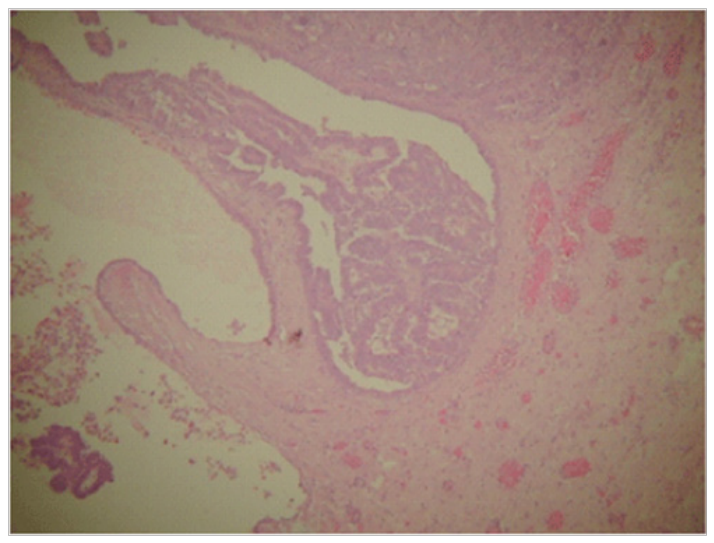

Figure I Fallopian tube carcinoma.

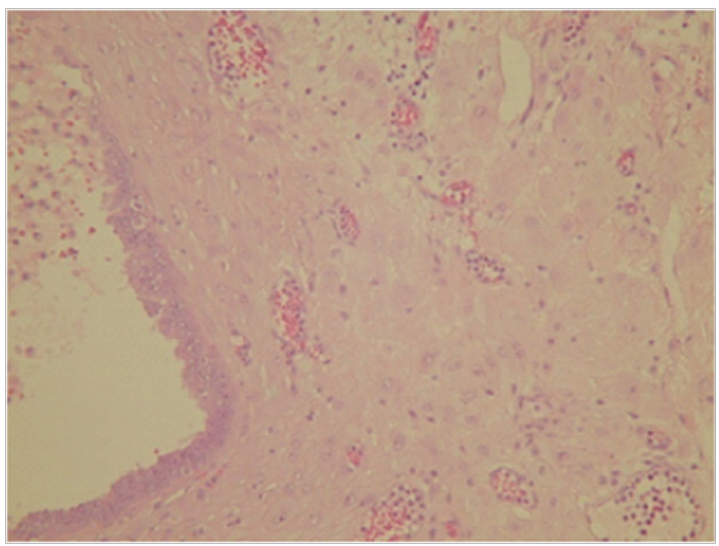

Figure 2 Progression of normal fallopian tube epithelium into dysplasia and then malignant transformation.

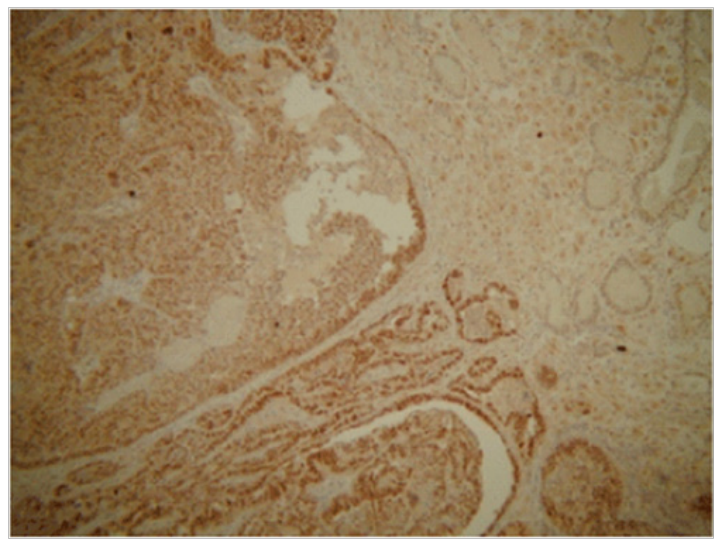

Figure 3 ER expression of the tumour. The tumour cells show strong positivity for ER receptors.
The patient's postoperative recovery was complicated by subacute small bowel obstruction secondary to pelvic haematoma, which required a second laparotomy to release the loops of small bowel. Also subclavian venous thrombosis was diagnosed which required anticoagulation therapy. She received supplementary total parenteral nutrition to help her recovery. She was discharged home well and received adjuvant chemotherapy.

\section{Discussion}

Cancer in pregnancy is rare and occurs in about 1:1000 pregnancies. The more common cancers in pregnancy are those that are more common in young people, including cervical cancer, breast cancer, Hodgkin's lymphoma, malignant melanoma and thyroid cancer. In hospital-based series, $0.2-2 \%$ of pregnancies are complicated by an adnexal mass, and approximately 1-6\% of these masses are malignant. ${ }^{4}$

The aetiology of PFTC is poorly understood; Riska et $a .^{5}$ suggested smoking as possible risk factor, also the group highlighted a possible genetic aetiology in view of an excess of colorectal and breast cancers after PFTC. PFTC is often presented with non-specific symptoms. In $50-60 \%$ of cases the presenting complaint is bleeding per vaginum, spotting or a pelvic mass, while $30-40 \%$ present with colicky or dull abdominal pain. Only $15 \%$ of cases present with Latzke triad of symptoms, which includes serosanguinous vaginal discharge, abdominal and pelvic pain.

The diagnosis of PFTC is usually made on histopathological examinations. The most common histology types of fallopian tube cancer are serous and endometrioid adenocarcinomas accounting for $66 \%$ and $29 \%$ respectively. ${ }^{6}$ Staging for fallopian tube is usually carried out during surgery. Pre-operative radiological staging is limited because of the pregnancy. The MRI scan reliably defines the nature of the adnexal mass, however in pregnancy; the fetus limits satisfactory imaging of the retroperitoneal space.

During pregnancy, CA-125 levels are elevated, with wide fluctuations in the first trimester. The levels in the early first trimester (five to eight weeks) are particularly high, with a mean of 55.8 and median of 36.2 (range, 6.9-251.2) $\mathrm{U} / \mathrm{ml}$. The levels then drop and remain below $35 \mathrm{U} / \mathrm{mL}$ through the rest of pregnancy (including immediately prior to delivery) only to rise again soon after delivery, with a mean of 39.8 and median of 41.9 (range, 10.7-296.7) $\mathrm{U} / \mathrm{ml}$. This level returns to normal after delivery in 2-10 weeks. ${ }^{7}$

Ca125 is expressed by PFTC and EOC and is raised in $80 \%$ of patients' pre-operatively particularly in patients with advanced or recurrent cancer. ${ }^{7}$ Gadducci et al. $^{8}$ showed that Ca125>35U/ml were found in $94.7 \%$ of stage III-IV disease and $68.7 \%$ of stage I-II disease. Significantly elevated Ca125 levels are therefore a valuable indicator of malignancy, even in pregnancy. PFTC has a higher rate of distal and retroperitoneal metastases. Retroperitoneal nodal metastasis is documented in $33 \%$ of women at all stages. ${ }^{9}$ The prognosis in pregnancy is often the same as for another woman of the same age with the same type and stage of cancer. If the response to chemotherapy is favorable, the prognosis is slightly more optimistic but remains guarded. ${ }^{10}$

In our case the presenting complain was threatening preterm labour at 31 weeks of gestation. Planned delivery at 34 weeks 
provided optimal outcome for the fetus. At laparotomy the tumour was confined to the pelvis with no obvious palpable lymphadenopathy and was optimally debulked. The diagnosis of fallopian tube cancer was made on histopathological examination. This did not alter the patient's surgical treatment and management, which is similar to the presumed diagnosis of EOC. Metastatic spread was present; however this was confined to the pelvis, with positive cytology making it a FIGO Stage IIB PFTC. We believe delivery at 34 weeks optimised the baby's outcome without significant detriment to the mother's health.

Although the patient had immediate postoperative complications, this has not delayed her adjuvant treatment with chemotherapy. She underwent treatment with the combination Carboplatin - Paclitaxel chemotherapy regime. The efficacy of chemotherapy treatment was monitored by serial measurements of the Ca125 levels which decreased significantly.

\section{Conclusion}

Fallopian tube cancers are rare and difficult to diagnose and often present at advanced stage. This woman's presentation with premature contractions led to an ultrasound and vaginal examination and to the discovery of a pelvic mass. This precipitated a series of investigations that led to the diagnosis of a malignant tumour with a detrimental impact on the mother and fetus. The chemotherapy regime used was effectively monitored by CA 125 . The authors urge clinicians to be vigilant in investigating suspicious pelvic masses in pregnancy with ultrasound and $\mathrm{Ca} 125$.

\section{Acknowledgments}

None.

\section{Conflicts of interest}

The authors declare there is no conflict of interests.

\section{References}

1. Kalampokas E, Sofoudis C, Boutas I, et al. Primary fallopian tube carcinoma: a case report and mini-review of the literature. Eur J Gynaecol Oncol. 2014;35(5):595-596.

2. Woolas RP, Smith JHF, Sarharnis P, et al. Fallopian tube carcinoma: an under-recognised primary neoplasm. Int J Gynecol Cancer. 1997;7(4):284288.

3. Chung C, Lee M, Chu P, et al. Case report: Occurrence of fallopian tube cancer in a patient with previous history of estrogen receptor positive breast cancer. Biosci Trends. 2009;3(5):200-201.

4. Leiserowitz GS, Xing G, Cress R, et al. Adnexal masses in pregnancy: How often are they malignant? Gynecol Oncol. 2006;101(2):315-321.

5. Riska A, Pukkala E, Scelo G, et al. Second primary malignancies in females with primary fallopian tube cancer. Int J Cancer. 2007;120(9):2047-2051.

6. Ajithkumar TV, Minimole AL, John MM, et al. Primary fallopian tube carcinoma. Obstet Gynecol Surv. 2005;60(4):247-252.

7. Spitzer M, Kaushal N, Benjamin F. Maternal CA-125 levels in pregnancy and the puerperium. J Reprod Med. 1998;43(4):387-392.

8. Gadducci A, Landoni F, Sartori E, et al. Analysis of treatment failures and survival of patients with fallopian tube carcinoma: a cooperation task force (CTF) study. Gynecol Oncol. 2001;81(2):150-159.

9. Tamimi HK, Figge DC. Adenocarcinoma of the uterine tube: potential for lymph node metastases. Am J Obstet Gynecol. 1981;141(2):132-137.

10. Peters WA $3^{\text {rd }}$, Andersen WA, Hopkins MP, et al. Prognostic features of carcinoma of the fallopian tube. Obstet Gynecol. 1988;71(5):757-762. 